\title{
A detached stellar-mass black hole candidate in the globular cluster NGC 3201
}

\author{
Benjamin Giesers, ${ }^{1 \star}$ Stefan Dreizler,${ }^{1} \dagger$ Tim-Oliver Husser, ${ }^{1}$ Sebastian Kamann,,${ }^{1,2}$ \\ Guillem Anglada Escudé, ${ }^{3}$ Jarle Brinchmann, ${ }^{4,5}$ C. Marcella Carollo, ${ }^{6}$ \\ Martin M. Roth, ${ }^{7}$ Peter M. Weilbacher, ${ }^{7}$ Lutz Wisotzki ${ }^{7}$ \\ ${ }^{1}$ Institut für Astrophysik, Georg-August-Universität Göttingen, Friedrich-Hund-Platz 1, 37077 Göttingen, Germany \\ ${ }^{2}$ Astrophysics Research Institute, Liverpool John Moores University, 146 Brownlow Hill, Liverpool L3 5RF, United Kingdom \\ ${ }^{3}$ School of Physics and Astronomy, Queen Mary University of London, 327 Mile End Road, London, United Kingdom \\ ${ }^{4}$ Leiden Observatory, Leiden University, P.O. Box 9513, 2300 RA, Leiden, The Netherlands \\ ${ }^{5}$ Instituto de Astrofísica e Ciências do Espaço, Universidade do Porto, CAUP, Rua das Estrelas, PT4150-762 Porto, Portugal \\ ${ }^{6}$ Institute for Astronomy, Swiss Federal Institute of Technology (ETH Zurich), CH-8093 Zurich, Switzerland \\ ${ }^{7}$ Leibniz-Institut für Astrophysik Potsdam (AIP), An der Sternwarte 16, 14482 Potsdam, Germany
}

Accepted XXX. Received YYY; in original form ZZZ

\begin{abstract}
As part of our massive spectroscopic survey of 25 Galactic globular clusters with MUSE, we performed multiple epoch observations of NGC 3201 with the aim of constraining the binary fraction. In this cluster, we found one curious star at the mainsequence turn-off with radial velocity variations of the order of $100 \mathrm{~km} \mathrm{~s}^{-1}$, indicating the membership to a binary system with an unseen component since no other variations appear in the spectra. Using an adapted variant of the generalized Lomb-Scargle periodogram, we could calculate the orbital parameters and found the companion to be a detached stellar-mass black hole with a minimum mass of $(4.36 \pm 0.41) \mathrm{M}_{\odot}$. The result is an important constraint for binary and black hole evolution models in globular clusters as well as in the context of gravitational wave sources.
\end{abstract}

Key words: stars: black holes - techniques: imaging spectroscopy - techniques: radial velocities - binaries: spectroscopic - globular clusters: individual: NGC 3201

\section{INTRODUCTION}

Owing to their old ages and high masses, Galactic globular clusters probably have produced a large number of stellarmass black holes during their lifetimes. Nevertheless, there is an ongoing debate about the number of black holes that actually remain in the cluster. In the absence of continuous star formation, stellar-mass black holes soon become the most massive objects in the cluster, where they accumulate around the centres as a consequence of mass segregation. However, because of the high mass-ratio with respect to the surviving low-mass stars ( $\gtrsim 4: 1)$, it is expected that the black holes form a dense nucleus that is decoupled from the dynamics of the remaining cluster (Spitzer 1969). Interactions within this nucleus are then expected to eject the majority of black holes, so that only few survive after $1 \mathrm{Gyr}$ (Kulkarni et al. 1993; Sigurdsson \& Hernquist 1993).

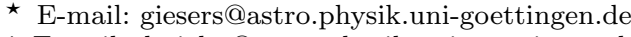

$\dagger$ E-mail: dreizler@astro.physik.uni-goettingen.de
However, over the past years, radio observations have revealed several sources in extragalactic and Galactic globular clusters that are likely to be stellar-mass black holes according to their combined radio and X-ray properties (Maccarone et al. 2007; Strader et al. 2012; Chomiuk et al. 2013). Under the assumption that only a small fraction of the existing black holes are actively accreting matter from a companion (Kalogera et al. 2004), these detections point to much richer black hole populations in globular clusters than previously thought. In fact, state-of-the art models for clusters do predict that the retention fractions of black holes might be significantly enhanced compared to the earlier studies mentioned above (e.g. Breen \& Heggie 2013; Morscher et al. 2013). The reason for this is that the Spitzer (1969) instability only develops partially and the black hole nucleus does not detach from the kinematics of the remaining cluster. As a consequence, the evaporation time-scale is prolonged.

The search for black holes in globular clusters has recently gained further importance through the first detection of gravitational waves, produced by the coalescence of two 
Table 1. Barycentric corrected radial velocity $v_{\mathrm{r}}$, (Vega) magnitude $I_{\mathrm{F} 814 \mathrm{~W}}$, and seeing (See.) measurements for the target star. ( $\triangle$ JD: Julian observation date JD-2456978 d, ESO ID: ESO Programme ID Code.)

\begin{tabular}{|c|c|c|c|c|}
\hline$\overline{\Delta \mathrm{JD}[\mathrm{d}]}$ & $\overline{v_{\mathrm{r}}[\mathrm{km} / \mathrm{s}]}$ & $\overline{I_{\mathrm{F} 814 \mathrm{~W}}[\mathrm{mag}]}$ & See. ["] & ESO prog. ID \\
\hline 0.83733 & $570.8 \pm 2.2$ & $16.88 \pm 0.08$ & 0.82 & 094.D-0142 \\
\hline 11.85794 & $512.9 \pm 2.7$ & $16.89 \pm 0.06$ & 0.90 & 094.D-0142 \\
\hline 11.86438 & $511.9 \pm 2.8$ & $16.91 \pm 0.08$ & 0.84 & 094.D-0142 \\
\hline 30.81091 & $475.0 \pm 2.2$ & $16.83 \pm 0.06$ & 0.60 & 094.D-0142 \\
\hline 30.83250 & $482.1 \pm 2.4$ & $16.88 \pm 0.07$ & 0.66 & 094.D-0142 \\
\hline 31.78096 & $477.1 \pm 2.0$ & $16.86 \pm 0.06$ & 0.72 & 094.D-0142 \\
\hline 31.80265 & $480.8 \pm 2.1$ & $16.87 \pm 0.07$ & 0.88 & 094.D-0142 \\
\hline 149.49256 & $536.8 \pm 2.0$ & $16.90 \pm 0.06$ & 0.60 & 095.D-0629 \\
\hline 151.47767 & $550.8 \pm 3.6$ & $16.82 \pm 0.07$ & 1.12 & 095.D-0629 \\
\hline 153.47698 & $559.3 \pm 2.5$ & $16.86 \pm 0.08$ & 1.00 & 095.D-0629 \\
\hline 156.47781 & $585.4 \pm 2.2$ & $16.86 \pm 0.08$ & 1.04 & 095.D-0629 \\
\hline 160.47808 & $609.5 \pm 2.0$ & $16.87 \pm 0.08$ & 0.70 & 095.D-0629 \\
\hline 441.74475 & $476.2 \pm 2.0$ & $16.90 \pm 0.06$ & 0.66 & 096.D-0175 \\
\hline 441.76738 & $472.0 \pm 1.8$ & $16.88 \pm 0.07$ & 0.64 & 096.D-0175 \\
\hline 443.74398 & $474.8 \pm 2.1$ & $16.89 \pm 0.06$ & 0.66 & 096.D-0175 \\
\hline 443.76792 & $472.5 \pm 2.1$ & $16.85 \pm 0.08$ & 0.54 & 096.D-0175 \\
\hline 538.47410 & $471.1 \pm 1.9$ & $16.86 \pm 0.06$ & 0.80 & 097.D-0295 \\
\hline 542.47958 & $471.9 \pm 2.0$ & $16.88 \pm 0.06$ & 0.78 & 097.D-0295 \\
\hline 808.87270 & $501.4 \pm 2.7$ & $16.80 \pm 0.06$ & 0.60 & 098.D-0148 \\
\hline 809.87675 & $512.7 \pm 2.5$ & $16.87 \pm 0.08$ & 0.96 & 098.D-0148 \\
\hline
\end{tabular}

massive black holes (Abbott et al. 2016a). As shown by Abbott et al. (2016c) or Askar et al. (2017), dense star clusters represent a preferred environment for the merging of such black hole binaries. Hence, it would be crucial to overcome the current observational limits in order to increase our sample of known black holes. Compared to radio or Xray studies, dynamical searches for stellar companions have the advantages of also being sensitive to non-accreting black holes and of providing direct mass constraints. We are currently conducting a large survey of 25 Galactic globular clusters with MUSE (Multi Unit Spectroscopic Explorer, Bacon et al. 2010), which provides us with spectra of currently 600 to 27000 stars per cluster (see Kamann et al. 2017). Our survey includes a monitoring for radial velocity variations, which is very sensitive to the detection of stellar companions of massive objects (i.e. black holes, neutron stars and white dwarfs). Here, we report the detection of a $(4.36 \pm 0.41) \mathrm{M}_{\odot}$ black hole in the globular cluster NGC 3201.

\section{OBSERVATIONS AND DATA REDUCTION}

The observational challenge in globular clusters is the crowding resulting in severe blending of nearby stars especially in the cluster cores. For photometric measurements of dense globular clusters, instruments like the Hubble Space Telescope (HST) provide sufficient spatial resolution to obtain independent measurements of most of the stars (Sarajedini et al. 2007). For spectroscopic surveys, investigations of dense stellar fields were limited to the brightest stars or to regions with sufficiently reduced crowding. In globular clusters, the combination of field of view $\left(1^{\prime} \times 1^{\prime}\right)$ and spatial sampling $\left(300 \times 300\right.$ spaxel $\left.^{2}\right)$ of MUSE allows us to extract spectra with a spectral resolution of $1800<R<3500$ of some thousand stars per exposure (for more details see Husser et al. 2016). We use the standard ESO MUSE pipeline to reduce the MUSE raw data (Weilbacher et al. 2012). The

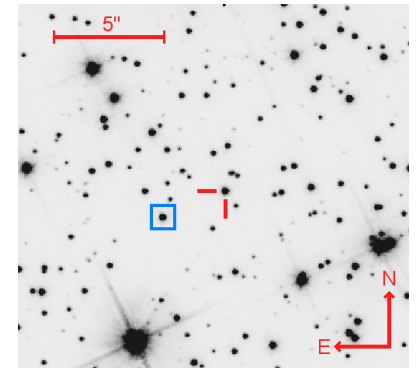

(a) HST

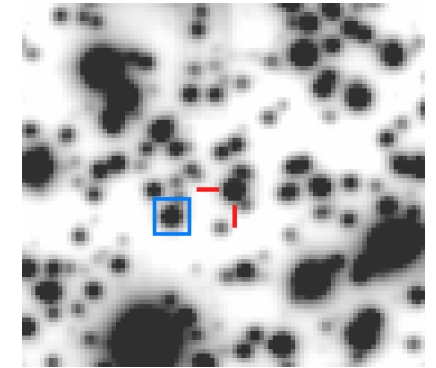

(b) MUSE
Figure 1. Charts with the target star marked with red crosshairs and the reference star marked within a blue square. (a) The image is taken from the HST ACS globular cluster survey from Sarajedini et al. (2007) and Anderson et al. (2008). (b) Same field of view seen by MUSE with a seeing of $0.6^{\prime \prime}$. The displayed image is a cut (90x81 pixels) from an integrated white light image of the MUSE data cube.

extraction is done with a PSF-fitting technique using the combined spatial and spectral information (Kamann et al. 2013, 2014).

This work is part of our investigation of the binarity of clusters using the radial velocity method. Binaries that are able to survive in the dense environment of a globular cluster are so tight (see Hut et al. 1992) that even HST is unable to resolve the single components such that they will appear as a single point-like source. Radial velocity surveys, however, will rather easily detect those compact binary systems. Except for the case when both stars have the same brightness, the extracted MUSE spectrum will be dominated by one of the stars, i.e. we mostly detect single line binary systems. With the knowledge of the stellar mass and the orbital parameters from our analyses, we can nevertheless infer the minimum masses of the unseen companions.

Up to this publication, we have observed three globular clusters NGC 104 (47 Tuc), NGC 3201, and NGC 5139 ( $\omega$ Cen) with sufficiently many observations to analyse the radial velocity signals $v_{\mathrm{r}}$ of individual stars. We identified three stars with radial velocity variations exceeding $100 \mathrm{~km} \mathrm{~s}^{-1}$. Only one of them (hereafter called target star) in NGC 3201 appears in two overlapping pointings, resulting in 20 extracted spectra with good signal-to-noise ratios (from 24 to 56,43 on average). The other two stars need more observations to analyse the orbital parameters. Table 1 lists the target star's derived radial velocities and seeing values for each observation from five observation runs.

\section{PHOTOMETRIC AND SPECTROSCOPIC ANALYSIS}

The investigation of the target star properties is performed in two steps. Photometry provides the mass of the target star and allows us to exclude alternative explanations for the large radial velocity signal. Below, we describe the spectral analysis from which we obtain the cluster membership. 


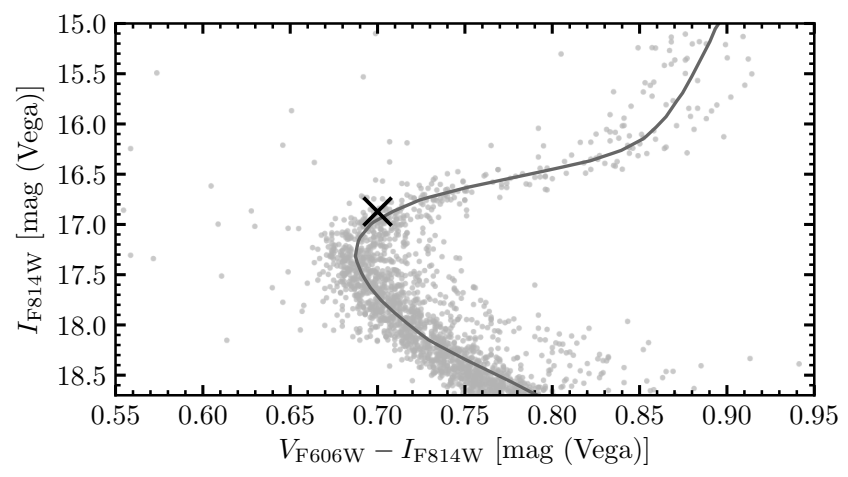

Figure 2. CMD of the globular cluster NGC 3201 created from the HST ACS photometry of Anderson et al. (2008). The target star position is highlighted by a black X. The line represents our best-fitting PARSEC isochrone (Bressan et al. 2012, for more details see subsection 3.1).

\subsection{Photometric analysis}

Figure 1 shows the target star (red crosshairs) with a magnitude of $I_{\mathrm{F} 814 \mathrm{~W}}=16.87 \mathrm{mag}(\mathrm{Vega})$ and its nearby stars from HST ACS data (Anderson et al. 2008). To monitor the reliability of our analyses, we have picked the star in the blue square with a magnitude of $I_{\mathrm{F} 814 \mathrm{~W}}=17.11$ as a reference star $\left(\mathrm{RA}=10^{\mathrm{h}} 17^{\mathrm{m}} 37^{\mathrm{s}} .36\right.$, Dec. $\left.=-46^{\circ} 24^{\prime} 56^{\prime \prime} 48\right)$. The three nearest stars have magnitudes fainter than 19 mag. For a consistency check and for investigating photometric variability, we convolve each flux calibrated spectrum with the corresponding ACS filter function and compare it to the ACS photometry. For all extracted spectra, we reach a magnitude accuracy of at least 95 per cent compared to the HST ACS magnitude (see Table 1). This indicates that the target star is extracted without contamination.

In order to derive the mass and surface gravity $\log g$ for the spectrum fit (see Table 2) of the target star, we compare its HST ACS colour and magnitude with a PARSEC isochrone (Bressan et al. 2012). For the globular cluster NGC 3201, we found the best matching isochrone compared to the whole HST ACS Colour-magnitude diagram (CMD) with the isochrone parameters $[\mathrm{M} / \mathrm{H}]=-1.39 \mathrm{dex}$ (slightly above the comparable literature value $[\mathrm{Fe} / \mathrm{H}]=-1.59 \mathrm{dex}$, Harris 1996), age $=11 \mathrm{Gyr}$, extinction $E_{B-V}=0.26$, and distance $=4.8 \mathrm{kpc}$ (see Fig. 2). The target star is at the main-sequence turn-off with a mass of $(0.81 \pm 0.05) \mathrm{M}_{\odot}$ as estimated from the isochrone.

Although the position of the target star in the CMD is not in the classical instability strip, we want to exclude radial velocity variations caused by photometric variability (e.g. due to pulsations). Therefore, we use the differential photometry method on our MUSE data. We first run an iterative algorithm to find a large sample of reference stars (which are present in all observations). After these stars have been identified, normally with an intrinsic standard deviation of $0.005 \mathrm{mag}$ in the filter $I_{\text {Bessel }}$ reconstructed from the extracted spectrum, we compare each star of each observation with our reference stars selecting 20 with comparable colour. The photometric analysis for the target star shows no significant variation (see Table 1 ). We would expect significant changes in the brightness of the target star to ex-

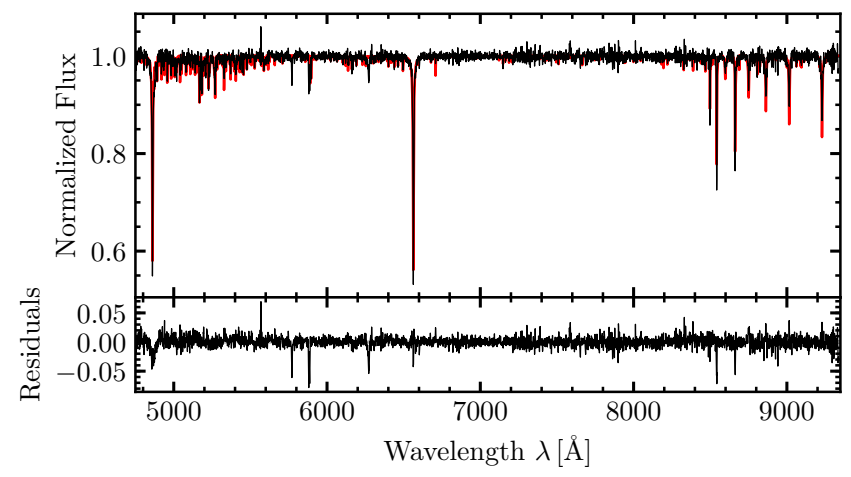

Figure 3. The combination of all radial velocity corrected spectra of the target star (in black). The best-fitting PHOENIX spectrum is indicated in the background in red (thicker curve for better visibility). The bottom panel shows the residuals after subtracting the best fit to the data.

plain such a high radial velocity amplitude via pulsations. We therefore conclude that the radial velocity variations are not intrinsic to the target star. The absence of large photometric variations also suggests the absence of an interacting binary. We like to note that no radio or X-ray source is known at the target star's position. For instance, Strader et al. (2013) performed a deep systematic radio continuum survey for black holes also in NGC 3201, but did not publish any discovery. Some X-ray sources were found by Webb et al. (2006), but none at the target star's coordinates.

\section{$3.2 \quad$ Spectroscopic analysis}

After the extraction of point sources, the individual spectra of the 20 visits are fitted against our Göttingen Spectral Library (Husser et al. 2013) of synthetic PHOENIX spectra to determine the stellar effective temperature and metallicity as well as radial velocities and the telluric absorption spectrum. The simultaneous fit of stellar and telluric spectra is performed with a least-squares minimization comparing the complete observed spectrum against our synthetic spectra. The telluric absorptions are used to correct for small remaining wavelength calibration uncertainties, allowing us to reach a radial velocity precision down to $1 \mathrm{~km} \mathrm{~s}^{-1}$. For more details about the stellar parameter fitting methods, we refer to Husser et al. (2013, 2016). We find huge changes in radial velocity between the spectra of up to $138 \mathrm{~km} \mathrm{~s}^{-1}$. We got the same variation if we do just cross-correlations between the spectra and the initial template, of course with higher uncertainties. Besides the radial velocity signal, the spectral fitting of the individual spectra did not reveal any other significant variations. The radial velocities are given in Table 1, whereas the mean stellar parameters are given in Table 2.

Using the derived radial velocities, the individual spectra are placed into rest frame and are then combined using the drizzling method from Fruchter \& Hook (2002) and finally normalized to the continuum. The combined spectrum in Fig. 3 is compared to one of our PHOENIX spectra for a star at the main-sequence turn-off in the globular cluster NGC 3201. The spectral properties match the position of the star in the CMD displayed in Fig. 2. We note that there 
Table 2. Target star properties. The position and magnitude were taken from the HST ACS globular cluster survey catalogue (Anderson et al. 2008), mass and surface gravity are derived from isochrone fitting, effective temperature and metallicity are derived from spectral fitting. For more details see Sect. 3.

\begin{tabular}{rlrl}
\hline \hline RA & $10^{\mathrm{h}} 17^{\mathrm{m}} 37^{\mathrm{s}} .090$ & Dec. & $-46^{\circ} 24^{\prime} 55^{\prime \prime} 31$ \\
$I_{\mathrm{F} 814 \mathrm{~W}}$ & $16.87 \pm 0.02 \mathrm{mag}($ Vega $)$ & & \\
$M$ & $(0.81 \pm 0.05) \mathrm{M}_{\odot}$ & $\log g$ & $(3.99 \pm 0.05) \mathrm{dex}$ \\
$T_{\mathrm{eff}}$ & $(6126 \pm 20) \mathrm{K}$ & {$[\mathrm{M} / \mathrm{H}]$} & $(-1.50 \pm 0.02) \mathrm{dex}$ \\
\hline
\end{tabular}

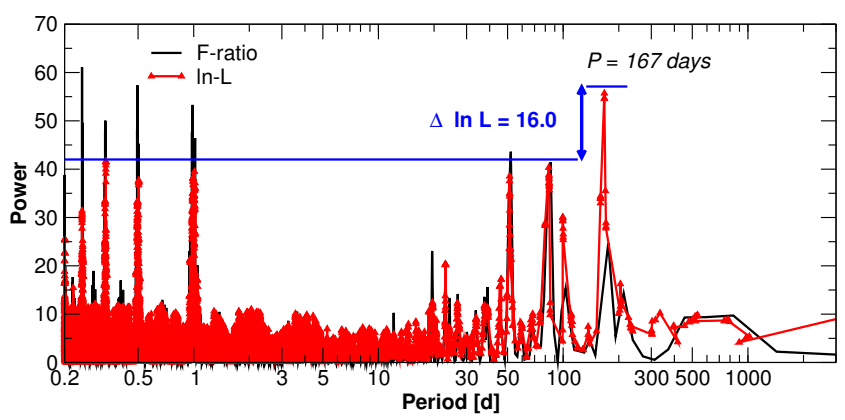

Figure 4. Likelihood periodogram of the radial velocities of the target star. The black curve is a version of the generalized LombScargle periodogram for circular orbits using the F-ratio statistic to represent the significance of each solution. The red triangles show the improvement on the log-likelihood statistic using a full Keplerian fit at the period search level instead.

are no emission lines that could indicate, for example, a cataclysmic variable or a compact binary with an illuminated low-mass star and a hot companion like a white dwarf or neutron star.

The mean radial velocity $(506 \pm 1) \mathrm{km} \mathrm{s}^{-1}$ and mean metallicity $[\mathrm{M} / \mathrm{H}]=(-1.50 \pm 0.02)$ dex of the target star is in good agreement with the cluster parameters in the Harris (1996) catalogue. Further, the fitted radial velocity of the binary barycentre (see Table 3) matches precisely the radial velocity of the cluster $(494.0 \pm 0.2) \mathrm{km} \mathrm{s}^{-1}$. This makes the target star a bonafide cluster member.

\section{RESULTS}

The analysis of the radial velocity variation is done using the generalized Lomb-Scargle (GLS) periodogram (Zechmeister \& Kürster 2009), the likelihood function approach of Baluev (2008), and a final fit of a Keplerian orbit. Figure 4 shows the likelihood periodogram of the target star for the period range $0.2-1000 \mathrm{~d}$. The black curve (F-ratio) represents the GLS periodogram for circular orbits. It shows highly significant periods at $1 \mathrm{~d}$, fractions of $1 \mathrm{~d}, 51 \mathrm{~d}$, and $83 \mathrm{~d}$. The $1 \mathrm{~d}$ period and fractions of it are aliases of our nightly observation basis. With Keplerian fits for the same period range, the resulting picture is different. The red triangles (ln- $\mathrm{L}$ curve) show these Keplerian fits as a log-likelihood statistic. The $167 \mathrm{~d}$ period has a very low false-alarm probability of $2.2 \times 10^{-8}$, so the signal is extremely significant. Compared to all other peaks, the ln-likelihood of the preferred solution is higher by $\Delta \ln L=16$. Within the framework of this type of periodogram, this implies a $\sim 8.9 \times 10^{6}$ higher probability.
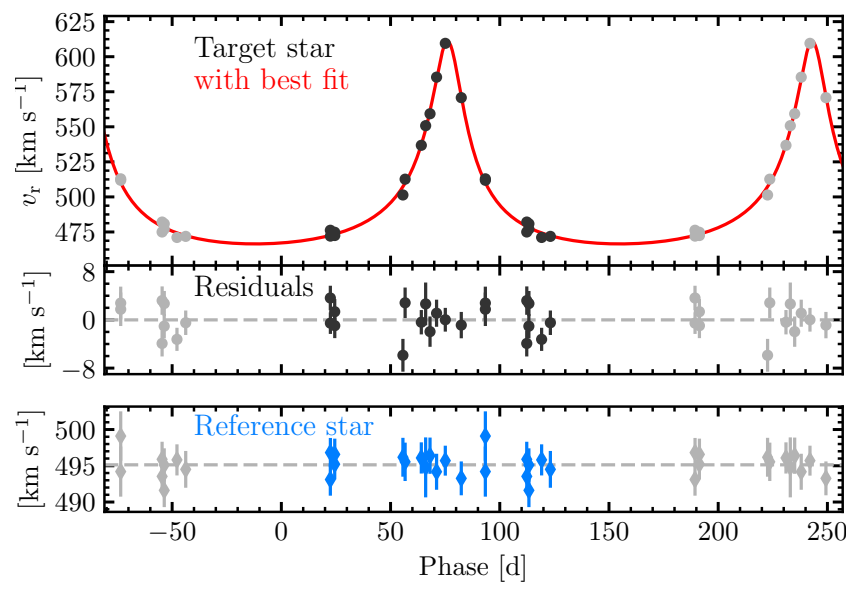

Figure 5. The top panel shows the radial velocity measurements $v_{\mathrm{r}}$ of the target star, phase folded for the $167 \mathrm{~d}$ period. Error bars are smaller than the data points. The red curve shows the bestfitting Keplerian orbit (see Table 3). The middle panel contains the residuals after subtracting this best-fitting model to the data. The bottom panel shows the radial velocity measurements of the reference star. Grey dots are phase shifted duplicates of the black ones, and are included to improve the visualization.

Table 3. Binary system properties. The Keplerian parameters were calculated with an MCMC consisting of $10^{7}$ iterations. The derived mass was calculated using $2.0 \times 10^{4}$ MCMC samples and the same number of target star mass samples.

\begin{tabular}{rl} 
Period $P$ & $166.88_{-0.63}^{+0.71} \mathrm{~d}$ \\
\hline \hline Doppler semi-amplitude $K$ & $(69.4 \pm 2.5) \mathrm{km} \mathrm{s}^{-1}$ \\
Eccentricity $e$ & $0.595 \pm 0.022$ \\
Argument of periastron $\omega$ & $(2.6 \pm 3.2)^{\circ}$ \\
Periastron passage $T_{0}$ & $(57140.2 \pm 0.5) \mathrm{d}$ \\
Barycentric radial velocity $\gamma_{0}$ & $(494.5 \pm 2.4) \mathrm{km} \mathrm{s}^{-1}$ \\
Linear trend $\dot{\gamma}$ & $(-0.27 \pm 2.70) \mathrm{km} \mathrm{s}^{-1}$ \\
Jitter $s$ & $0.68_{-0.25}^{+0.40} \mathrm{~km} \mathrm{~s}^{-1}$ \\
\hline Minimum companion mass $M \sin (i)$ & $(4.36 \pm 0.41) \mathrm{M}_{\odot}$ \\
Minimum semi-major axis $a(M)$ & $(1.03 \pm 0.03) \mathrm{au}$ \\
\hline
\end{tabular}

Moreover, the $51 \mathrm{~d}$ and $83 \mathrm{~d}$ peaks are most likely only harmonics of this period $(83 \mathrm{~d} \approx 167 \mathrm{~d} / 2)$ and the window function, which has a high power at $135 \mathrm{~d}(1 / 51-1 / 83 \approx 1 / 135)$. We also performed a detection probability test comparable to Fiorentino et al. (2010) to verify that our sampling is sensitive to all periods in the probed range.

Figure 5 shows the radial velocity measurements phase folded with the $167 \mathrm{~d}$ period and the best-fitting Keplerian orbit solution. The reduced $\chi^{2}$ of the Keplerian orbit fit is 1.2 (for comparison the reduced $\chi^{2}$ of the best circular orbit at the $83 \mathrm{~d}$ period is 45$)$. The resulting orbital parameters fitted with the MCMC approach of Haario et al. (2006) are shown in Table 3 . The reference star does not show any significant radial velocity variation (reduced $\chi^{2}=0.42$ ).

\section{DISCUSSION AND CONCLUSIONS}

The data show strong evidence that the target star is in a binary system with a non-luminous object having a minimum mass of $(4.36 \pm 0.41) \mathrm{M}_{\odot}$. This object should be degenerate, 
since it is invisible and the minimum mass is significantly higher than the Chandrasekhar limit $\left(\sim 1.4 \mathrm{M}_{\odot}\right)$. The small distance of the system from the centre of the globular cluster $\left(10.8^{\prime \prime}\right)$ is expected from mass segregation. Most likely, the degenerate object has exceeded the Tolman-OppenheimerVolkoff limit that predicts all objects to collapse into black holes above $\sim 3 \mathrm{M}_{\odot}$ (Bombaci 1996). We note that the mass estimate of the dark companion depends only weakly on the mass of the target star within reasonable error estimates (e.g. for the unrealistic case of a target star with $0.2 \mathrm{M}_{\odot}$, the minimum companion mass will still be above $3 \mathrm{M}_{\odot}$ ).

Alternatively, the discovery could eventually be explained through a triple star system that consists of a compact double neutron star binary with a main-sequence turnoff star around it. In the literature, neutron star binaries show a narrow mass distribution of $(1.35 \pm 0.04) \mathrm{M}_{\odot}$ per star (Thorsett \& Chakrabarty 1999; Lattimer 2012). Recent discoveries show that a single neutron star could reach $2.0 \mathrm{M}_{\odot}$ (Özel \& Freire 2016). Thus, a double neutron star system with both components having more than $2.0 \mathrm{M}_{\odot}$ could explain the observations. Since such a system was not observed to date and the actual mass of the discovered object is probably higher, a black hole scenario is more likely. In this case, our results represent the first direct mass estimate of a (detached) black hole in a globular cluster.

The recent discovery of coalescing black hole binaries (Abbott et al. 2016b) suggests that there is a large population of stellar-mass binary black holes in the Universe. Our results confirm that the components of such binaries can be found in globular clusters.

The black hole is assumed to be detached because the closest possible approach in our best-fitting model is 0.4 au and the Roche limit for the target star with a reasonable radius of $1 R_{\odot}$ is of the order of $3 R_{\odot}$. We have no evidence that the black hole accretes mass emits X-rays or radio jets.

Compared to other globular clusters, the most unusual structural parameter of NGC 3201 is the large cluster core radius (1.3', see Harris 1996). As the presence of black holes can lead to an expansion of the core radius through interactions between black holes and stars (Strader et al. 2012), the discovery of the presented black hole could be an indication that NGC 3201 possesses an extensive black hole system in its core. More observations with MUSE could reveal more black hole companions using the radial velocity method.

To get the true system mass function, it is necessary to measure the inclination of the system. Since the Sun's distance to the globular cluster NGC 3201 is $4.9 \mathrm{kpc}$ (Harris 1996), the orbital movement of the target star is of the order of 0.2 mas. This should be observable with interferometers. Unfortunately, for the ESO interferometer VLTI GRAVITY, a reference star with K $11 \mathrm{mag}$ within $2^{\prime \prime}$ is missing (Gillessen et al. 2010). Maybe it could be observable with diffraction limited large telescopes like HST, the VLT with NACO at UT1, or the VLT with the new adaptive optics facility (GRAAL) at UT4. Certainly, this would be a nice astrometry task for the JWST and for the upcoming first-light instrument MICADO at the ELT (Massari et al. 2016).

\section{ACKNOWLEDGEMENT}

BG, SD, SK, and PMW acknowledge support from the German Ministry for Education and Science (BMBF Verbundforschung) through grants 05A14MGA, 05A17MGA, 05A14BAC, and 05A17BAA. GAE acknowledges a GaussProfessorship granted by the Akademie für Wissenschaften zu Göttingen. JB acknowledges support by Fundação para a Ciência e a Tecnologia (FCT) through national funds (UID/FIS/04434/2013) and Investigador FCT contract IF/01654/2014/CP1215/CT0003, and by FEDER through COMPETE2020 (POCI-01-0145-FEDER-007672). This research is supported by the German Research Foundation (DFG) with grants DR 281/35-1 and KA 4537/2-1. Based on observations made with ESO Telescopes at the La Silla Paranal Observatory (see programme IDs in Table 1). Based on observations made with the NASA/ESA Hubble Space Telescope, obtained from the data archive at the Space Telescope Science Institute. STScI is operated by the Association of Universities for Research in Astronomy, Inc. under NASA contract NAS 5-26555. Supporting data for this article is available at: http://musegc.uni-goettingen.de.

This is a pre-copyedited, author-produced version of an article accepted for publication in Monthly Notices of the Royal Astronomical Society: Letters following peer review. The version of record, Volume 475, Issue 1, 21 March 2018, Pages L15-L19, is available online at: https://academic.oup.com/mnrasl/article/475/ 1/L15/4810643 (DoI: 10.1093/mnrasl/slx203).

\section{REFERENCES}

Abbott B. P., et al., 2016a, Physical Review Letters, 116, 061102 Abbott B. P., et al., 2016b, Physical Review Letters, 116, 241103 Abbott B. P., et al., 2016c, ApJ, 818, L22

Anderson J., et al., 2008, AJ, 135, 2055

Askar A., Szkudlarek M., Gondek-Rosińska D., Giersz M., Bulik T., 2017, MNRAS, 464, L36

Bacon R., et al., 2010, in Society of Photo-Optical Instrumentation Engineers (SPIE) Conference Series. p. 8, doi: $10.1117 / 12.856027$

Baluev R. V., 2008, MNRAS, 385, 1279

Bombaci I., 1996, A\&A, 305, 871

Breen P. G., Heggie D. C., 2013, MNRAS, 432, 2779

Bressan A., Marigo P., Girardi L., Salasnich B., Dal Cero C., Rubele S., Nanni A., 2012, MNRAS, 427, 127

Chomiuk L., Strader J., Maccarone T. J., Miller-Jones J. C. A., Heinke C., Noyola E., Seth A. C., Ransom S., 2013, ApJ, 777, 69

Fiorentino G., et al., 2010, ApJ, 708, 817

Fruchter A. S., Hook R. N., 2002, PASP, 114, 144

Gillessen S., et al., 2010, in Optical and Infrared Interferometry II. p. $77340 \mathrm{Y}$ (arXiv:1007.1612), doi:10.1117/12.856689

Haario H., Laine M., Mira A., Saksman E., 2006, "Statistics and Computing", 16, 339

Harris W. E., 1996, AJ, 112, 1487

Husser T.-O., Wende-von Berg S., Dreizler S., Homeier D., Reiners A., Barman T., Hauschildt P. H., 2013, A\&A, 553, A6

Husser T.-O., et al., 2016, A\&A, 588, A148

Hut P., et al., 1992, PASP, 104, 981

Kalogera V., King A. R., Rasio F. A., 2004, ApJ, 601, L171

Kamann S., Wisotzki L., Roth M. M., 2013, A\&A, 549, A71 


\section{L6 B. Giesers et al.}

Kamann S., Wisotzki L., Roth M. M., Gerssen J., Husser T.-O., Sandin C., Weilbacher P., 2014, A\&A, 566, A58

Kamann S., et al., 2017, preprint, (arXiv:1710.07257)

Kulkarni S. R., Hut P., McMillan S., 1993, Nature, 364, 421

Lattimer J. M., 2012, Annual Review of Nuclear and Particle Science, 62, 485

Maccarone T. J., Kundu A., Zepf S. E., Rhode K. L., 2007, Nature, 445,183

Massari D., et al., 2016, in Adaptive Optics Systems V. p. 99091G (arXiv: 1607.04412), doi:10.1117/12.2232478

Morscher M., Umbreit S., Farr W. M., Rasio F. A., 2013, ApJ, 763, L15

Özel F., Freire P., 2016, ARA\&A, 54, 401

Sarajedini A., et al., 2007, AJ, 133, 1658

Sigurdsson S., Hernquist L., 1993, Nature, 364, 423

Spitzer Jr. L., 1969, ApJ, 158, L139

Strader J., Chomiuk L., Maccarone T. J., Miller-Jones J. C. A., Seth A. C., 2012, Nature, 490, 71

Strader J., Miller-Jones J., Maccarone T., Chomiuk L., 2013, The ATCA Survey for Black Holes in Southern Globular Clusters, ATNF Proposal

Thorsett S. E., Chakrabarty D., 1999, ApJ, 512, 288

Webb N. A., Wheatley P. J., Barret D., 2006, A\&A, 445, 155

Weilbacher P. M., Streicher O., Urutia T., Jarno A., PécontalRousset A., Bacon R., Böhm P., 2012, in Software and Cyberinfrastructure for Astronomy II. p. 84510B, doi: $10.1117 / 12.925114$

Zechmeister M., Kürster M., 2009, A\&A, 496, 577

This paper has been typeset from a $\mathrm{T}_{\mathrm{E}} \mathrm{X} / \mathrm{LAT}_{\mathrm{E}} \mathrm{X}$ file prepared by the author. 